\title{
Online Mining (Recently) Maximal Frequent Itemsets over Data Streams
}

\author{
Hua-Fu Li ${ }^{\mathrm{a}}$, Suh-Yin Lee ${ }^{\mathrm{a}}$, Man-Kwan Shan ${ }^{\mathrm{b}}$ \\ ${ }^{a}$ Department of Computer Science and Information Engineering \\ National Chiao-Tung University, Hsinchu, Taiwan \\ hfli@csie.nctu.edu.tw, sylee@csie.nctu.edu.tw \\ ${ }^{b}$ Department of Computer Science, National Chengchi University, Taipei, Taiwan \\ mkshan@cs.nccu.edu.tw
}

\begin{abstract}
A data stream is a massive, open-ended sequence of data elements continuously generated at a rapid rate. Mining data streams is more difficult than mining static databases because the huge, high-speed and continuous characteristics of streaming data. In this paper, we propose a new one-pass algorithm called DSM-MFI (stands for Data Stream Mining for Maximal Frequent Itemsets), which mines the set of all maximal frequent itemsets in landmark windows over data streams. A new summary data structure called summary frequent itemset forest (abbreviated as SFIforest) is developed for incremental maintaining the essential information about maximal frequent itemsets embedded in the stream so far. Theoretical analysis and experimental studies show that the proposed algorithm is efficient and scalable for mining the set of all maximal frequent itemsets over the entire history of the data streams.
\end{abstract}

\section{Introduction}

In recent years, various data mining techniques have been explored in the literature. One of the most important data mining problems is mining maximal frequent itemsets from a large database [2, 6, 7, 14, 25]. The problem of mining maximal frequent itemsets was first proposed by Bayardo [6]. The problem is defined as follows. Let $\Psi=\left\{i_{1}, i_{2}, \ldots, i_{n}\right\}$ be a set of literals, called items. Let database $D B$ be a set of transactions, and the size of $D B$ is denoted by $|D B|$. A transaction $T$ with $m$ items is denoted by $T=\left\{x_{1}, x_{2}, \ldots, x_{m}\right\}$, such that $T \subseteq \Psi$. A $k$-itemset is a set with $k$ items and denoted by $\left(x_{1}, x_{2}, \ldots, x_{k}\right)$. The support of an itemset $X$ is the number of transactions containing $X$ as a subset divided by the total number of transactions in the database, i.e., $|D B|$, and denoted by $\sup (X)$. An itemset
$X$ is called frequent if $\sup (X) \geq$ minusp, where minusp is a user-defined minimum support threshold in the range of $[0,1]$. The set of all frequent itemsets is denoted by FI. A frequent itemset is called maximal if it is not a subset of any other frequent itemsets. The set of all maximal frequent itemsets is denoted by $M F I$. The problem aims to find out the set of all maximal frequent itemsets with support greater than the userdefined minimum support threshold.

Recently, database and data mining communities have focused on a new data model, where data arrives in the form of continuous streams. It is often refer to as data streams or streaming data. Many applications generate large amount of data streams in real time, such as sensor data generated from sensor networks, online transaction flows in retail chains, Web record and click-streams in Web applications, call records in telecommunications, performance measurement in network monitoring and traffic management, etc.

The problem of mining of data streams is different from mining static datasets in the following aspects [5]. First, each data element of stream should be examined at most once. Second, the memory usage in the process of mining data streams should be bounded even though new data elements are continuously generated from the streams. Third, each element in the stream should be processed as fast as possible. Fourth, the analytical outputs of the stream should be instantly available when the user requested. Finally, the errors of outputs should be constricted as small as possible. The processing model of data streams is shown in Figure 1. The continuous characteristic of streaming data makes it essential to use the algorithms which require only one scan over the stream for knowledge discovery. The huge nature of stream makes it impossible to store all the data into main memory or even in secondary storage. This motivates the design of summary data structure with small footprints that can support both one-time and continuous queries. In other words, one- 
pass data stream mining algorithms have to sacrifice the correctness in the analytical results by allowing some counting errors. Consequently, previous multiplepass algorithms studied for mining static datasets are not feasible for mining data streams.

With years of research into this research, several data stream mining problems have been discussed, such as frequent itemset mining [12, 8, 13, 20, 22], closed frequent structure mining [19], computing statistics [24], data clustering $[3,15,21]$, decision tree construction and data classification [1, 10, 16, 23], change detection and mining [12, 11, 17], regression analysis [9], Web click-stream mining [18], etc.

In this paper, we will focus on the problem of mining the set of all maximal frequent itemsets in landmark windows over data streams. The proposed algorithm DSM-MFI (stands for Data Stream Mining for Maximal Frequent Itemsets) is composed of four steps. First, it reads a block of transactions from the buffer in main memory, and sorts the items of transactions in the lexicographical order. Second, it constructs and maintains the in-memory summary data structure SFI-forest (stands for Summary Frequent Itemset forest). Third, it prunes the infrequent patterns from the summary data structure. Fourth, it searches the set of all maximal frequent itemsets from the current summary data structure. Steps 1 and 2 are performed in sequence for a new incoming data block. Steps 3 and 4 are usually performed periodically or when it is needed. The theoretical analysis and experimental results show that the proposed algorithm is efficient and scalable for mining the set of all maximal frequent itemsets over the entire history of the data streams.

The remainder of this paper is organized as follows. Problem definition is given in Section 2. Algorithm DSM-MFI to find the MFI is described in Section 3. The performance results are presented in Section 4. We discuss the sliding windows in Section 5. Section 6 concludes our study.

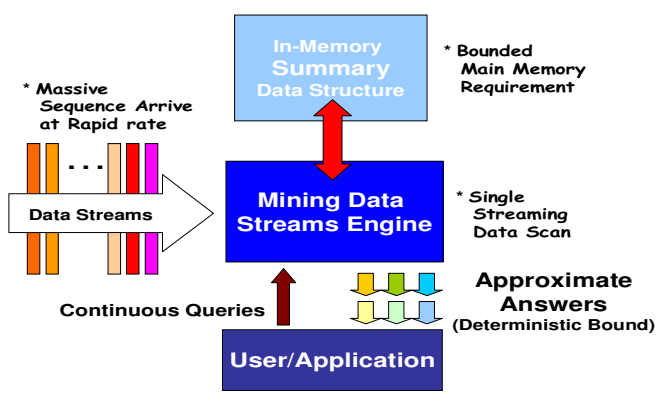

Figure 1. Processing model of data streams

\section{Problem definition}

Let $\Psi=\left\{i_{1}, i_{2}, \ldots, i_{n}\right\}$ be a set of literals, called items. A data stream, $D S=\left[\mathrm{W}_{1}, \mathrm{~W}_{2}, \ldots, \mathrm{W}_{N}\right)$, is an infinite sequence of basic windows, where each basic window $\mathrm{W}_{i}, \forall i=1,2, \ldots, N$, is associated with a window identifier $i$, and $N$ is the window identifier of the "latest" basic window $B_{N}$. A basic window consists of a fixed sized number of transactions, where each transaction is composed of a set of items (named itemset). The size of a basic window $\mathrm{W}$ is denoted by IWI. The current length (abbreviated as $C L$ ) of data stream is $\left|\mathrm{W}_{1}\right|+\left|\mathrm{W}_{2}\right|+\cdots+\left|\mathrm{W}_{N}\right|$. A transaction $T$ with $k$ items is denoted by $T=\left(x_{1}, x_{2}, \ldots, x_{k}\right)$, such that $T \subseteq$ $\Psi$. A $k$-itemset is an itemset with $k$ items and denoted by $\left(x_{1}, x_{2}, \ldots, x_{k}\right)$.

Because it is unrealistic to store all the data into limited main memory or even in secondary storage, the single-pass algorithm for mining data streams has to sacrifice the correctness of their analytical results by allowing some frequency errors. Therefore, the true support of an itemset $X$ is the number of transactions of the stream containing the itemset $X$ as a subset, and denoted by X.tsup. The estimated support of an itemset $X$ is the estimated true support stored in the summary data structure, and denoted by X.esup. Note that $1 \leq$ X.esup $\leq X$.tsup. The current length of data stream with respect to an itemset $X$ is $\left|\mathrm{W}_{j}\right|+\left|\mathrm{W}_{j+1}\right|+\cdots+\left|\mathrm{W}_{N}\right|$, where basic window $\mathrm{W}_{j}$ is the first window containing $X$ recorded in the current summary data structure, and is denoted by $X . C L$. In this paper, the itemsets embedded in the data streams can be divided into three types: frequent itemset, significant itemset, and infrequent itemset. An itemset $X$ is called frequent if X.esup $\geq$ $s \cdot X$.CL, where $s$ is a user-defined minimum support threshold in the range of $[0,1]$. An itemset $X$ is called significant if $s \cdot X . C L>X . e s u p \geq \varepsilon \cdot X . C L$, where $\varepsilon$ is a user-specified maximum support error threshold in the range of $[0, s]$. An itemset $X$ is called infrequent if $X . e s u p<\varepsilon \cdot X . C L$. An itemset is called maximal if it is not a subset of any other frequent itemsets.

In this paper, we focus on mining the set of all maximal frequent itemsets in landmark windows over data streams. In the landmark model, knowledge is discovered based on the values between a specific window identifier called landmark and the present window identifier. In this paper, the landmark is 1 , and it is an unrestricted window.

Consequently, given a data stream $D S=\left[\mathrm{W}_{1}\right.$, $\mathrm{W}_{2}, \ldots, \mathrm{W}_{N}$, a minimum support threshold $s$ in the range of $[0,1]$, and a maximum support error threshold 
$\varepsilon$ in the range of $[0, s]$, the problem of mining maximal frequent itemsets in landmark windows (landmark =1) is to find the set of all maximal frequent itemsets over the entire history of data streams.

\section{The proposed algorithm: DSM-MFI}

The proposed algorithm DSM-MFI is composed of four steps. First, it reads a window of transactions from the buffer in main memory, and sorts the items of transactions in a lexicographical order. Second, it constructs and maintains the in-memory summary data structure. Third, it prunes the infrequent information from the summary data structure. Fourth, it searches the maximal frequent itemsets from the current summary data structure. Steps 1 and 2 are performed in sequence for a new incoming basic window. Steps 3 and 4 are usually performed periodically or when it is needed.

\subsection{Constructing the summary data structure}

In this section, a new in-memory summary data structure called SFI-forest is defined, and an efficient algorithm is proposed to construct and maintain the summary data structure.

Definition 1 A Summary Frequent Itemset forest (abbreviated as SFI-forest) is an extended prefix treebased summary data structure defined below.

1. SFI-forest is composed of a frequent item list (abbreviated as FI-list), and a set of summary frequent itemset trees (abbreviated as SFI-trees) of item-suffixes, denoted by item-suffix.SFI-trees.

2. Each node in the item-suffix.SFI-tree consists of four fields: item-id, esup, window-id, and nodelink, where item-id is the item identifier of the inserting item, esup registers the number of transactions represented by a portion of the path reaching the node with the item-id, the value of window-id assigned to a new node is the window identifier of the current basic window, and nodelink links up a node with the next node with the same item-id in the same SFI-tree, or null if there is none.

3. Each entry in the FI-list consists of four fields: item-id, esup, window-id, and head of node-link (a pointer links to the root node with item-id of the SFI-tree), abbreviated as head-link, where item-id registers which item identifier the entry represents, esup records the number of transactions containing the item with the item-id, the value of window-id assigned to a new entry is the window identifier of current basic window, the head-link points to the root node of the item-id.SFI-tree. Note that each entry with item-id in the FI-list is an item-suffix and it is also the root node of the item-id.SFI-tree.

4. Each item-suffix.SFI-tree has a specific opposite frequent item list (abbreviated as OFI-list) with respect to the item-suffix, and denoted by itemsuffix.OFI-list. The item-suffix.OFI-list consists of four fields: item-id, esup, window-id, and headlink. The item-suffix.OFI-list operates the same as the FI-list except that the field head-link links to the first node carrying the item-id in the itemsuffix.SFI-tree. Note that $\mid$ item-suffix.OFI-list $\mid=$ $\mid F I$-list $\mid$ in the worst case, where $\mid F I$-list $\mid$ denotes the total number of entries in the current FI-list.

Figure 2 outlines the SFI-forest construction of the DSM-MFI algorithm. The constriction scenario of SFIforest is described as follows. First of all, DSM-MFI algorithm reads a transaction $T$ from the current basic window $\mathrm{W}_{N}$. Then, DSM-MFI projects the transaction $T$ into many sub-transactions, and inserts these subtransactions into the SFI-forest. The detail of projection is defined as follows. A transaction $T$ with $m$ items, i.e., $T=\left(x_{1}, x_{2}, \ldots, x_{m}\right)$, should be projected by inserting $m$ item-suffix sub-transactions into the current SFI-forest. In other words, the transaction is converted into $\mathrm{m}$ subtransactions; that is, $\left(x_{1}, x_{2}, \ldots, x_{m}\right),\left(x_{2}, x_{3}, \ldots, x_{m}\right), \ldots$, and $\left(x_{m}\right)$. These $m$ sub-transactions are called itemsuffix transactions, since the first item of each subtransaction is an item-suffix of the original transaction $T$. The step is called transaction projection, and denoted by Transaction-Projection $(T)=\left\{x_{1}\left|T, x_{2}\right| T, \ldots, x_{i} \mid T, \ldots\right.$, $\left.x_{m} \mid T\right\}$, where $x_{i} \mid T=\left(x_{i}, x_{i+1}, \ldots, x_{m}\right), \forall i=1,2, \ldots, m$. Note that the projection cost a transaction with $\mathrm{m}$ items fro constructing the summary data structure SFI-forest is $\left(m^{2}+m\right) / 2$, i.e., $m+(m-1)+\cdots+1$.

After performing the transaction projection of the incoming transaction $T$, DSM-MFI inserts the items of $T$ into the FI-list, and removes $T$ from the current window in the main memory. Then, the set of items of these item-suffix transactions are inserted into the itemsuffixes.SFI-forests as branches, and updates the estimated support of corresponding item-suffixes.OFIlists. If an itemset share a prefix with an itemset already in the SFI-tree, the new itemset will share a prefix of the branch representing that itemset. In addition, a counter of estimated support is associated with each node in the tree. The counter is updated when an itemsuffix transaction causes the insertion of a new branch. Figure 3 shows the subroutines of construction and maintenance of SFI-forest. 
Algorithm 1 (SFI-forest construction)

Input: A data stream, $D S=\left[\mathrm{W}_{1}, \mathrm{~W}_{2}, \ldots, \mathrm{W}_{N}\right)$, a user-specified minimum support threshold $s \in(0,1)$, and a user-defined maximum support error threshold $\varepsilon \in(0, s)$.

Output: A SFI-forest generated so far.

1: FI-list $=\{\} ; / *$ initialize the FI-list to empty.*/

2: foreach basic window $\mathrm{W}_{j}$ do $/ * j=1,2, \ldots, N *$ /

3: foreach transaction $T=\left(x_{1}, x_{2}, \ldots, x_{m}\right) \in \mathrm{W}_{j}(j=1,2, \ldots, N)$ do $/ * m \geq 1$ and $j$ is the current window identifier $* /$

foreach item $x_{i} \in T$ do $/ *$ the maintenance of FI-list $* /$

if $x_{i} \notin$ FI-list then

create a new entry of form $\left(x_{i}, 1, j\right.$, head-link $)$ into the

FI-list; $\quad{ }^{*}$ the entry form is (item-id, item-id.esup, window-id, head-link) */

7: $\quad$ else $/ *$ the entry already exists in the FI-list*/

8: $\quad x_{i}$.esup $=x_{i}$.esup +1

$/ *$ increment the estimated support of item-id $x_{i}$ by one*/

9: $\quad$ end if

10: end for

11: call Transaction-Projection $(T, j)$;

$l^{*}$ project the transaction with each item-suffix $x_{i}$ for constructing the $x_{i}$.SFI-tree $* /$

12: end for

13: call SFI-forest-pruning(SFI-forest, $\varepsilon, N)$;

14: end for

/* Step 3 of DSM-MFI algorithm */

Figure 2. Algorithm of SFI-forest Construction

Subroutine Transaction-Projection /* Step 2 of DSM-MFI */

Input: A transaction $T=\left(x_{1}, x_{2}, \ldots, x_{m}\right)$ and the current window-id $j$; Output: $x_{i}$ SFI-tree, $\forall i=1,2, \ldots, m$;

1: foreach item $x_{i}, \forall i=1,2, \ldots, m$, do

2: $\quad$ SFI-tree-maintenance $\left(\left[x_{i} \mid X\right], x_{i}\right.$.SFI-tree, $\left.j\right)$;

$I^{*} X=x_{1}, x_{2}, \ldots, x_{m}$ is the original incoming transaction $T * /$

3: end for

$/ *\left[x_{i} \mid X\right]$ is an item-suffix transaction with the item-suffix $x_{i} * /$

\section{Subroutine SFI-tree-maintenance /* Step 2 of DSM-MFI */}

Input: An item-suffix transaction $\left(x_{i}, x_{i+1}, \ldots, x_{m}\right)$, the current window-id $j$, and $x_{i}$.SFI-tree, $\forall i=1,2, \ldots, m$;

Output: A modified $x_{i}$.SFI-tree, $\forall i=1,2, \ldots, m$;

1: foreach item $x_{l}$ do $/ * l=i+1, i+2, \ldots, m * /$

if $x_{l} \notin x_{i}$. OFI-list then $/ * x_{i}$.OFI-list maintenance $* /$ create a new entry of form $\left(x_{l}, 1, j\right.$, head-link) into the $x_{i}$. OFI-list;

/*the entry form is (item-id, item-id.esup, window-id, head-link)*/

4: $\quad$ else $/ *$ the entry already exists in the $x_{i}$.OFI-list */

5: $\quad x_{l}$.esup $=x_{l}$.esup +1

$/ *$ increment the estimated support of item-id $x_{l}$ by one*/

6: end if

7: endfor

8: foreach item $x_{i}, \forall i=1,2, \ldots, m$, do $/ * x_{i}$. SFI-tree maintenance*/

9: $\quad$ if SFI-tree has a child node with item-id $y$ such that $y . i t e m-i d=$ $x_{i}$ item-id then

10: $\quad$ y.esup $=$ y.esup $+1 ; / *$ increment $y$ 's estimated support by $1 * /$

11: else create a new node of the form $\left(x_{i}, 1, j\right.$, node-link);

$I^{*}$ initialize the estimated support of the new node to 1 , and link its parent link to SFI-tree, and its node-link linked to the nodes with same item-id via the node-link structure. */

12: end if
13: end for

Subroutine SFI-forest-pruning /* Step 3 of DSM-MFI */

Input: A SFI-forest, a maximum support error threshold $\varepsilon$, and the current window identifier $N$;

Output: A SFI-forest which contains the set of all significant and frequent itemsets.

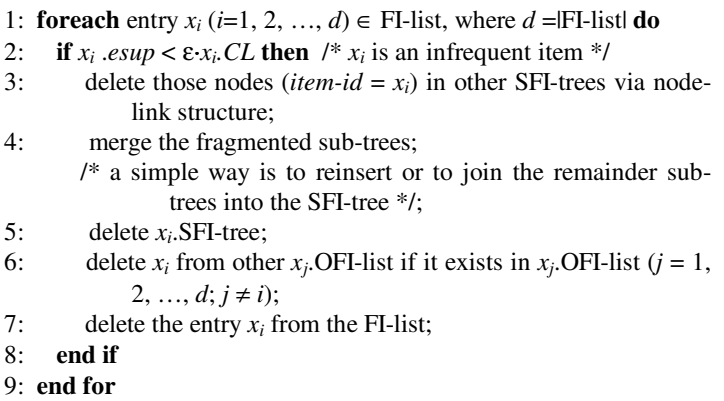

Figure 3. Subroutines of SFI-forest construction algorithm

\subsection{Pruning infrequent items from SFI-forest}

According to the Apriori principle [4]: if any length $k$ pattern is not frequent, its length $(k+1)$ superpatterns can never be frequent, only the frequent 1itemsets are used to construct candidate itemsets in the next pass. Thus, the set of itemsets stored in the current summary data structure containing the infrequent items is pruned. The pruning mechanism is usually performed periodically or when it is needed.

Let the maximum support error threshold be $\varepsilon$ in the range of $[0, s]$, where $s$ is a user-specified minimum support threshold in the range of $[0,1]$. The pruning method of summary data structure of DSM-MFI is that a 1-itemset $X$ and its supersets are deleted from the current SFI-forest if X.esup $<\varepsilon \cdot X$.CL, where $X$ is an entry of FI-list. For each entry of the form (item-id, esup, window-id, head-link) in the FI-list, if its itemid.esup is less than the threshold $\varepsilon \cdot i t e m-i d . C L$, it can be regarded as an infrequent item. At this time, three operations are performed in sequence. First, DSM-MFI deletes the item-id.OFI-list, item-id.SFI-tree, and the entry with item-id from the FI-list. Second, DSM-MFI removes the infrequent item with item-id from other OFI-lists by traversing the FI-list. Third, DSM-MFI deletes the infrequent item with item-id from other SFItrees, and reconstructs these SFI-trees by reinserting these modified item-suffix transactions. After pruning all infrequent items from SFI-forest, SFI-forest contains the set of frequent itemsets and significant itemsets of the data stream so far. 
The next step of DSM-MFI is to determine the set of all maximal frequent itemsets from the SFI-forest constructed so far. The step is performed only when the analytical results of the stream is requested.

\subsection{Determining maximal frequent itemsets from the current summary data structure}

Once FI-list, containing all frequent items of the stream generated so far, is constructed, DSM-MFI can derive the set of all maximal frequent itemsets by traversing the SFI-forest based on the Apriori principle. In this paper, an efficient mechanism called top-down selection of Maximal Frequent Itemsets (abbreviated as todoMFI) is proposed to mine the set of all maximal frequent itemsets from the current SFI-forest. It is especially useful at mining long frequent itemsets. The todoMFI algorithm is shown in Figure 4, and is described as follows.

Assume that there are $k$ frequent 1-itemsets, such as $e_{1}, e_{2}, \ldots, e_{k}$, in the current FI-list, and each item $e_{i}, \forall i$ $=1,2, \ldots, k$, has a $e_{i}$.OFI-list. Note that the size of $e_{i}$.OFI-list is denoted by $\mid e_{i}$.OFI-listl, and the items, namely $o_{1}, o_{2}, \ldots, o_{j}$, within the $e_{i}$. OFI-list are denoted by $e_{i} . o_{1}, e_{i} . o_{2}, \ldots, e_{i} . o_{j}$, respectively, where the value of $j$ is $\mid e_{i}$.OFI-listl. For each entry $e_{i}, \forall i=1,2, \ldots, k$, in the FI-list, DSM-MFI first generates a candidate maximal frequent $(j+1)$-itemset, i.e., $\left(e_{i}, e_{i} . o_{1}, e_{i} . o_{2}, \ldots, e_{i} . o_{j}\right)$, by combining the item-suffix $e_{i}$ and all the frequent items in $e_{i}$. OFI-list. Then, DSM-MFI uses the following traversal scheme to count its estimated support.

First, DSM-MFI starts with a specific frequent item $e_{i} . o_{l}(1 \leq l \leq j)$, whose estimated support is smallest, and traverses the paths containing the item with $e_{i} . o_{l}$ via node-links of $e_{i}$.SFI-tree to count the estimated support of the candidate $\left(e_{i}, e_{i} . o_{1}, e_{i} . o_{2}, \ldots, e_{i} . o_{j}\right)$. After that, if the estimated support is greater than or equal to the threshold $s \cdot e_{i} \cdot C L$, it is a maximal frequent itemset. Hence, all subsets of this maximal frequent itemset are also frequent itemsets, but not maximal frequent itemsets, according to the Apriori principle and the definition of maximal frequent itemset. On the other hand, if the estimated support of this candidate is less the threshold $s \cdot e_{i} \cdot C L$, it is not a frequent itemset. Hence, DSM-MFI uses the same mechanism to test all the subsets of the $(j+1)$-itemset using a level-by-level order until the testing of candidate maximal frequent 3itemsets. Only testing at 3 -itemsets is because all frequent 2 -itemsets can be generated by combining the item $e_{i}$ and the frequent 1 -itemsets of $e_{i}$.OFI-list. Note that a $(j+1)$-itemset can be decomposed into $C(j+1, j) j$ itemsets.
Algorithm 2 (top-down selection of Maximal Frequent Itemsets)

Input: A current SFI-forest, the current window identifier $N$, a minimum support threshold $s$, and a maximum support error threshold $\varepsilon$.

Output: A set of all maximal frequent itemsets.

1: $\quad$ MFI $_{\mathrm{temp}}$-list $=\varnothing ; \quad I^{*} \mathrm{MFI}_{\mathrm{temp}}$-list is a temporary list used to store the set of maximal frequent itemsets */

: foreach entry $e$ in the current FI-list do

do construct a candidate maximal frequent itemset $E$ with size $|E| \quad|*| E|=1+| e$. OFI-list $\mid * /$

count E.esup by traversing the $e$.SFI-tree;

if $E$.esup $\geq s \cdot E$. CL then

if $E \not \subset \mathrm{MFI}_{\mathrm{temp}}$-list and $E$ is not a subset of any other frequent itemsets in $\mathrm{MFI}_{\mathrm{temp}}$-list then

add $E$ into the MFI $_{\text {temp }}$-list;

remove $E$ 's subsets from the $\mathrm{MFI}_{\mathrm{temp}}$-list;

end if

else $/ *$ if $E$ is not a frequent itemset */

enumerate $E$ into itemsets with size $|E|-1$;

end if

until todoMFI finds the set of all maximal frequent itemsets with respect to entry $e$;

14: end for

Figure 4. Algorithm description of todoMFI

\subsection{Theoretical analysis}

In this section, we discuss the maximal estimated support error of maximal frequent itemsets generated by DSM-MFI algorithm, and the upper bound of space usage of a prefix tree-based summary data structure.

\subsubsection{Maximal estimated support error analysis}

Let X.window-id be the window-id of itemset $X$ stored in the current SFI-forest, the size of basic window be $k$ transactions, the maximum support error threshold be $\varepsilon$, and the current window-id of the data stream be window-id $(N)$. Now, we have the following theorem of maximal estimated support error guarantee of maximal frequent itemsets generated by DSM-MFI algorithm.

Theorem 1 X.tsup - X.esup $\leq \varepsilon \cdot(X$. window-id -1$) \cdot k$.

Proof: We prove by induction. Base case (X.window$i d=1):$ X.tsup $=$ X.esup. Thus, X.tsup - X.esup $\leq \varepsilon$. $(X$. window-id -1$) \cdot k$.

Induction step: Consider an itemset of the form $(X$, X.esup, X.window-id) that get deleted for some window-id $(N)>1$. The itemset was inserted in the SFIforest when window-id $(N+1)$ was being processed. The itemset $X$ whose $X$.window-id is window-id(N+1) in the FI-list could possibly have been deleted as late as the time when X.esup $\leq \varepsilon \cdot($ window-id $(N+1)-X$.window- 
$i d+1) \cdot k$. Therefore, X.tsup of itemset $X$ when that deletion occurred was no more than $\varepsilon \cdot($ window-id $(N+1)$ $-X$. window-id+1) $k$. Furthermore, the value X.esup is the estimated true support of $X$ since it was inserted. It follows that X.tsup which is the true support of $X$ in first basic window containing $X$ though the current window, is at most X.esup $+\varepsilon \cdot(\operatorname{window}-i d(N)-1) \cdot k$. Thus, we have X.tsup $-X$. esup $\leq \varepsilon \cdot(X$.window-id -1$) \cdot k$. As a result, DSM-MFI generates no false negative.

\subsubsection{Upper bound of space usage for constructing a prefix tree-based summary data structure}

In this section, we discuss the upper bound of space usage for constructing a prefix tree-based summary data structure.

Theorem 2 A prefix tree-based summary data structure has at most $2^{k}$ nodes for storing the set of all frequent itemsets of data streams, where $k$ is the number of frequent 1-itemsets generated so far.

Proof: Let $k$ be the number of frequent 1-itemsets in landmark windows over data streams generated so far. Hence, the number of potential frequent itemsets is $\mathrm{C}(k$, 1) regarding one item, $\mathrm{C}(k, 2)$ regarding two items, ..., $\mathrm{C}(k, i)$ regarding $i$ items, $\ldots$, and $\mathrm{C}(k, k)$ regarding $k$ items according to the Apriori heuristic. In a prefix tree-based summary data structure, an itemset is represented by a path and its support is maintained in the last node of the path. Thus, there are $\mathrm{C}(k, 1)$ nodes in the first level, $\mathrm{C}(k, 2)$ nodes in the second level, ..., $\mathrm{C}(k, i)$ nodes in the $i$ th level, $\ldots$, and $\mathrm{C}(k, k)$ nodes in the $k$ th level. There are totally $\mathrm{C}(k, 1)+\mathrm{C}(k, 2)+\cdots+$ $\mathrm{C}(k, i)+\cdots+\mathrm{C}(k, k)$ nodes in the prefix tree-based summary data structure. Consequently, the space upper bound of a prefix-tree based summary data structure is $O\left(2^{k}\right)$

\section{Performance evaluation}

The simulation model of our experimental studies is described in Section 4.1. The required resources, i.e., memory usage and execution time, of DSM-MFI are evaluated in Section 4.2. Notice that, we do not compare DSM-MFI algorithm with other algorithms [2, $6,7,14,25]$ in Section 4.2, since these approaches need at least two database scans for mining maximal frequent itemsets. It is not feasible for mining data streams.

\subsection{Simulation model}

All the experiments are performed on a $1 \mathrm{GHz}$ IBM $\mathrm{X} 24$ with $384 \mathrm{MB}$, and the program is written in Microsoft Visual C++ 6.0. The parameters of synthetic data generated by IBM synthetic data generator [4] are described as follows.

IBM synthetic datasets: T10.I5.D1000K and T30.I20.D1000K. The first synthetic dataset $T 10 . I 5$ has average transaction size $T$ of 10 items and the average size of frequent itemset $I$ is 5-items. It is a sparse dataset. In the second dataset T30.I20, the average transaction size $T$ and average frequent itemset size $I$ are set to 30 and 20, respectively. It is a dense dataset. Both synthetic datasets have 1,000,000 transactions. In the experiments, the synthetic data stream is broken into basic windows with size $50 \mathrm{~K}$ for simulating the continuous characteristic of streaming data, where $1 K$ denotes 1,000 . Hence, there are total 20 windows in these experiments. Moreover, the default value of user-defined minimum support threshold $s$ is $0.1 \%$, and the maximum support error threshold $\varepsilon$ is $0.1 \cdot s$, i.e., $0.01 \%$.

\subsection{Scalability study of DSM-MFI}

In this section, two primary factors, execution time and memory usage, are examined for mining the set of all maximal frequent itemsets in landmark windows over data streams, since both should be bounded online as time advances. Therefore, in Figure 5, the execution time grows smoothly as the dataset size increases from $2,000 \mathrm{~K}$ to $10,000 \mathrm{~K}$. The memory usage in Figure 6 for both synthetic datasets is stable as time progresses, indicating the scalability and feasibility of algorithm DSM-MFI. Notice that, the synthetic data stream used in Figure 6 is broken into 20 basic windows with size $50 \mathrm{~K}$ for simulating the continuous characteristic of data streams.

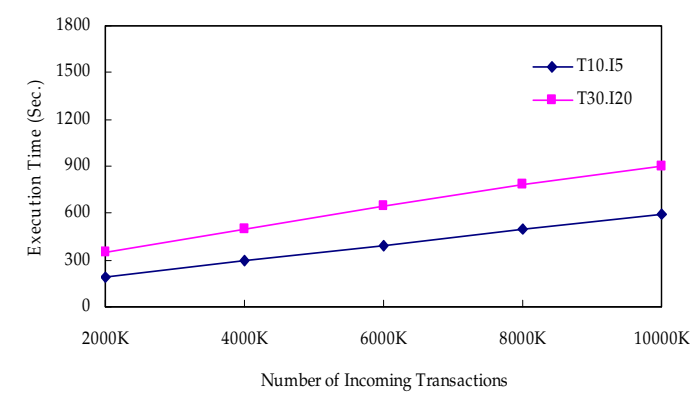

Figure 5. Required resources (execution time) of DSMMFI for IBM synthetic datasets: T10.I5 vs. T30.I20 


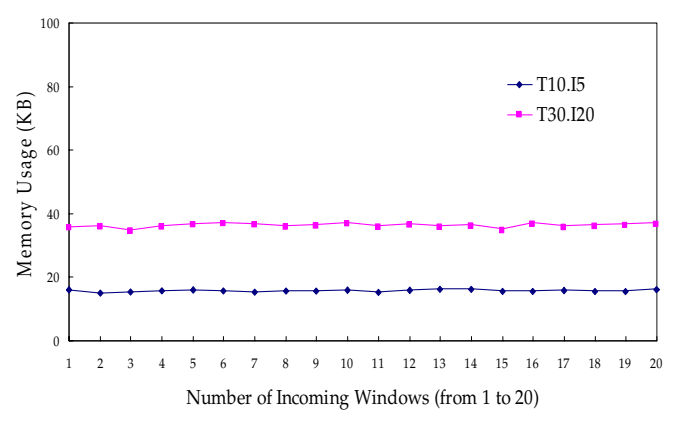

Figure 6. Required resources (Memory Usage) of DSM-MFI from window $\mathrm{W}_{1}$ to window $\mathrm{W}_{20}$

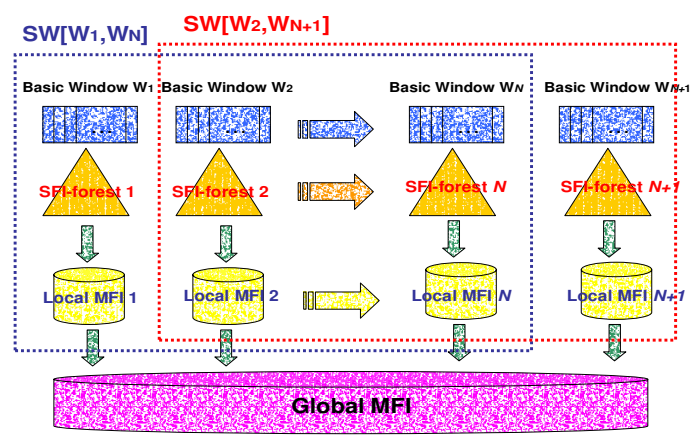

Figure 7. Mining model of DSM-RMFI

\section{Discussions}

In this section, we will discuss sliding window-based mining of maximal frequent itemsets over data streams.

\subsection{Sliding Window Mining over Data Streams}

In the sliding window model, the system stores only the $N$ most recent basic windows, $\mathrm{SW}\left[\mathrm{W}_{i+1}, \mathrm{~W}_{i+N}\right]$. The main issue is that as a new basic window arrives, oldest window must be removed from the main memory and their contribution discarded from the answer.

A DSM-MFI-based algorithm called DSM-RMFI (stands for Data Stream Mining for Recently Maximal Frequent Itemsets) is proposed to mine the set of all maximal frequent itemsets in sliding windows over data streams. The mining model of DSM-RMFI algorithm is shown in Figure 7. For each basic window $\mathrm{W}_{i}, \forall i=1$, $2, \ldots, N$, DSM-RMFI uses DSM-MFI algorithm to construct a SFI-forest $i$, and to find local maximal frequent itemsets (denoted by local MFI $i$ ) from the SFI-forest $i$. All local MFI $i, \forall i=1,2, \ldots, N$, are stored in a queue data structure. A list of global MFI, called
Global MFI, is used to store the set of all local maximal frequent itemset from basic windows $\mathrm{W}_{1}$ though $\mathrm{W}_{N}$. DSM-RMFI only maintains a queue of local MFIs and a Global MFI into main memory. The set of all maximal frequent itemsets (global MFI) are generated by searching the Global MFI.

When a new basic window with window-id $N+1$ arrives, DSM-RMFI removes the local MFI 1 from the front of the queue of local MFIs and subtracts the support of the local MFI 1 from the global MFI stored in the Global MFI. After that, DSM-RMFI uses DSMMFI algorithm to mine the set of all local maximal frequent itemsets from the new incoming basic window. Then, DSM-RMFI stores these local maximal frequent itemsets generated from the new window into local MFI $N+1$, and increases the support of global MFI, if the local maximal frequent itemsets already exist in the Global MFI. Otherwise, these new local maximal frequent itemsets are inserted into the Global MFI.

\section{Conclusions}

In this paper, we proposed a new, single-pass algorithm called DSM-MFI which mines the set of all maximal frequent itemsets over the entire history of the streaming data. In the DSM-MFI algorithm, a new inmemory summary data structure called SFI-forest is constructed for storing the frequent and significant itemsets of the streaming data generated so far. An efficient pattern selection method is developed to find the set of all maximal frequent itemsets from the current SFI-forest. Experiments with synthetic data datasets show that DSM-MFI is efficient on both sparse and dense datasets, and scalable to very long data streams. Moreover, a DSM-MFI-based sliding window mining algorithm is developed to mine the set of all maximal frequent itemsets in sliding windows over data streams.

\section{Acknowledgements}

The authors thank the reviewers' precious comments for improving the quality of the paper. The research is supported by National Science Council of R.O.C. under grant no. NSC93-2213-E-009-043.

\section{References}

[1] C.C. Aggarwal. A Framework for Diagnosing Changes in Evolving Data Streams. In ACM SIGMOD, 2003. 
[2] R. Agrawal, C. Aggarwal and V. Prasad. A Tree Projection Algorithm for Generation of Frequent Itemsets. Journal of Parallel and Distributed Computing, 2001.

[3] C. C. Aggarwal, J. Han, J. Wang, and P.S. Yu. A Framework for Clustering Evolving Data Streams. In Proc. of the $29^{\text {th }}$ VLDB conference, 2003.

[4] R. Agrawal and R. Srikant. Fast Algorithms for Mining Association Rules. In Conf. of the $20^{\text {th }} V L D B$ conference, pages 487-499, 1994.

[5] B. Babcock, S. Babu, M. Datar, R. Motwani, and J. Widom. Models and Issues in Data Stream Systems. In Proc. of the 2002 ACM Symposium on Principles of Database Systems (PODS 2002), ACM Press, 2002.

[6] Roberto Bayardo. Efficiently Mining Long Patterns from Databases. In ACM SIGMOD Conference, 1998.

[7] D. Burdick, M. Calimlim, and J. Gehrke. MAFIA: A Maximal Frequent Itemset Algorithm for Transactional Databases. In International Conference on Data Engineering, Apr. 2001.

[8] J. Chang and W. Lee. Finding Recent Frequent Itemsets Adaptively over Online Data Streams. In Proc. of the $9^{\text {th }}$ ACM SIGKDD International Conference \& Data Mining (KDD-2003), 2003.

[9] Y. Chen, G. Dong, J. Han, B. W. Wah, and J. Wang. Multi-Dimensional Regression Analysis of Time-Series Data Streams. In Proceedings of 2002 International Conference on Very Large Data Bases (VLDB'02), Hong Kong, China, Aug. 2002.11.

[10] P. Domingos and G. Hulten. Mining High-Speed Data Streams. In Proc. of the ACM Conference on Knowledge and Data Discovery (SIGKDD), 2000.

[11] G. Dong, J. Han, L.V.S. Lakshmanan, J. Pei, H. Wang and P.S. Yu. Online Mining of Changes from Data Streams: Research Problems and Preliminary Results. In Proceedings of the 2003 ACM SIGMOD Workshop on Management and Processing of Data Streams, June 2003.

[12] V. Ganti, J. Gehrke, and R. Ramakrishnan. Mining Data Streams under Block Evolution. SIGKDD Exploration, 3(2):1-10, Jan. 2002.

[13] C. Giannella, J. Han, J. Pei, X. Yan and P. S. Yu. Mining Frequent Patterns in Data Streams at Multiple Time Granularities. In Proc. of the NSF Workshop on Next Generation Data Mining, 2002.
[14] K. Gouda and M. Zaki. Efficiently Mining Maximal Frequent Itemsets. In Proc. of the IEEE International Conference on Data Mining, 2001.

[15] S. Guha, N. Mishra, R. Motwani, and L. O'Callaghan. Clustering Data Streams. In Proc. of the Annual Symp. on Foundations of Computer Science (FOCS), 2000.

[16] G. Hulten, L. Spencer, and P. Domingos. Mining Time-Changing Data Streams. In Proc. of the ACM Conference on Knowledge Discovery and Data Mining (SIGKDD), 2001.

[17] H.-F. Li and S.-Y. Lee. Single-Pass Algorithms for Mining Frequency Change Patterns with Limited Space in Evolving Append-only and Dynamic Transaction Data Streams. In IEEE International Conference on eTechnology, e-Commerce and e-Service (EEE-04), Mar. 2004.

[18] H.-F. Li, S.-Y. Lee and M.-K. Shan. On Mining Web-Click Streams for Path Traversal Patterns. In Proc. of the Thirteen International World Wide Web Conference (WWW-04), New York, May 2004.

[19] H.-F. Li, S.-Y. Lee and M.-K. Shan. Mining Frequent Closed Structures in Streaming Melody Sequences. In IEEE International Conference on Multimedia and Expo (ICME-2004), June 2004.

[20] G. S. Manku and R. Motwani. Approximate Frequency Counts Over Data Streams. In Proc. of the $28^{\text {th }}$ VLDB conference, 2002.

[21] L. O'Callaghan, N. Mishra, A. Meyerson, S.Guha, and R. Motwani. High-Performance Clustering of Streams and Large Data Sets. In Proc. of the 2002 International Conference of Data Engineering (ICDE), 2002.

[22] W.G. Teng, M.-S. Chen, and P. S. Yu. A RegressionBased Temporal Pattern Mining Scheme for Data Streams. In Proc. of the $29^{\text {th }}$ VLDB Conference, 2003.

[23] H. Wang, W. Fan, P. S. Yu, and J. Han. Mining Concept-Drifting Data Streams using Ensemble Classifiers. In ACM SIGKDD, 2003. Y. Zhu and D. Shasha. StatStream: Statistical Monitoring of Thousands of Data Streams in Real Time. In Proc. 28th Int. Conf. on Very Large Data Bases, 2002.

[24] Q. Zou, W. Chu, and B. Lu. SmartMiner: A Depth First Algorithm Guided by Tail Information for Mining Maximal Frequent Itemsets. In Proc. of the IEEE International Conference on Data Mining, 2002. 